Itinéraires Itinéraires

Littérature, textes, cultures

\title{
Cuisine : seuils et corridors
}

\section{Abdelkrim Chiguer}

\section{OpenEdition}

\section{Journals}

Édition électronique

URL : http://journals.openedition.org/itineraires/1565

DOI : 10.4000/itineraires. 1565

ISSN : 2427-920X

Éditeur

Pléiade

Édition imprimée

Date de publication : 1 novembre 2011

Pagination : 151-160

ISBN : 978-2-296-55720-8

ISSN : 2100-1340

Référence électronique

Abdelkrim Chiguer, «Cuisine : seuils et corridors », Itinéraires [En ligne], 2011-3 | 2011, mis en ligne le 01 novembre 2011, consulté le 01 mai 2019. URL : http://journals.openedition.org/itineraires/1565 : DOI : 10.4000/itineraires. 1565

\section{(c) (1) $\Theta$}

Itinéraires est mis à disposition selon les termes de la licence Creative Commons Attribution - Pas d'Utilisation Commerciale - Pas de Modification 4.0 International. 


\section{Cuisine : seuils et corridors}

\section{Abstract}

The work of the novelist, essayist and poet Abdelwahab Meddeb unfolds since Talismano (Paris, Christian Bourgois, 1978) until Aya dans les villes (Montpellier, Fata Morgana, 1999), according to the logic of a thematic and generic hybridity where words and words are the traces of an archaic and contemporary Maghreb memory. For this novelist, the kitchen is the place of a conception work and "preparation" of a recipe and a cure against anemia and/or amnesia.

Keywords : body, Abdelwahab Meddeb, Morocco, culinary, hybridity Mots clés : corps, Abdelwahab Meddeb, Maroc, culinaire, hybridité

Les mets que le gourmand choisit avec amour et dispose avec art ne sont pas loin d'être comme des mots, faits de couleurs et de parfums, de souvenirs précis et d'un profond dépôt de mémoire involontaire. [...] Cosmonaute du goût et de la vie, le gourmand bien servi prend une fusée, pour un voyage paradoxal (parce qu'il est en aller-retour) dans un espace non euclidien, c'est-à-dire, non plat, mais un espace courbe et fuselé où les lignes parallèles finissent quelque part par se croiser et permettent, comme dans le cas d'une carte routière rageusement chiffonnée, de faire se toucher des choses très éloignées l'une de l'autre.

(Grégoire Polet, Petit éloge de la gourmandise, Paris, Gallimard, 2010, p. 79 et 91)

Depuis son roman, Talismano ${ }^{1}$, l'« œuvre » d'Abdelwahab Meddeb s'inscrit dans un contexte de revigoration du passé, de la mémoire, là où la cuisine forme une « pièce » des plus privilégiées dans le processus de quête des « lieux qui ouvrent des questions en terres d'Islam² ». En effet, à l'affût d'adresses à même de lui permettre de « vérifier »l'hypothèse d'une matière

1. Abdelwahab Meddeb, Talismano, Paris, Christian Bourgois, 1978.

2. Abdelwahab Meddeb, «Les fins de l'étranger », Dédale, n 9-10, 1999, p. 11-17. 
à haute densité originelle, l'œuvre, réécriture autant qu'autoréécriture, agit toujours liée à un atelier de type expérimental composé de trois matériaux qui, « frottés » les uns aux autres, permettent sinon d'illustrer, d'authentifier ladite épaisseur, du moins, d'être et d'agir lors de chaque visite, revisite, au plus près de sa texture. Les trois pôles de l'atelier à l'œuvre renvoient à un " héros écrivain ${ }^{3}$ ", souvent accompagné de son double : Aya, à des fragments d'espaces : rosace, hammam, chambre ou cuisine, et à des fragments d'avant-textes, tous genres et toutes substances confondus : versets, dits prophétiques, aphorismes nietzschéens, photographies, films et peintures. Depuis Talismano jusqu'au recueil de récits-essais intitulé : Aya dans les villes ${ }^{4}$, la cuisine est parmi les lieux les plus visités, revisités. Composé de textes autant que de paratextes, notre corpus se déploie en liant l'avant (Talismano) et l'après (Talismano bis), et ce, en vertu d'une série de répétitions et de variations permettant de voir s'édifier, peu à peu, une cuisine au sens d'une " place » arborescente troublant la transparence d'un corpus de recettes, celles là mêmes qui s'évertuent à orienter le corps, son palais et ses papilles. Notre approche consiste à décliner, déplier, ce même toujours autre d'une " place baroque ${ }^{5}$ " passant outre les seuils et les frontières d'un espace dogmatique et rhétorique rétif aux risques de tout geste et de toute dépense dits inutiles et qui, au nom d'une logique, en grande partie, fonctionnelle, utilitaire, voire « stomacale ", procède à la " dépréciation de la générosité qui va de pair avec le discrédit de la poésie, et la promotion de l'avarice s'accompagn[ant] implicitement d'une valorisation de la $\operatorname{prose}^{6}{ }^{»}$. Des principes tels que : Stiââb (assimilation), Hâdm (digestion), ou encore Al-Hifzî 'an'dahrî'kalb (apprentissage par cœur), en disent long sur la nature de cette non-place, non-lieu réduisant l'Adab, la littérature, à un acte d'observation et de respect des normes de la bonne conduite. La critique littéraire « classique », régie par une catégorie comme : Mîzân (balance) ou Muwâzana (pondération et modération), ne manque pas, quant à elle, d'œuvrer pour écarter le risque de tout « don » excessif, a-gramm(es)-atical. Régie par le fiduciaire, la même rhétorique préfère,

3. Formule de Claude Ollier dont l'hérö̈sme est d'agir là où « ça » fait lieu, ou mieux : mi-lieux entre « espaces, souvenirs et écrits » et, notamment, dans les « régions mitoyennes » où il « ressent fortement des liens d'espace ». Claude Ollier, Liens d'espaces, Paris, POL, 2000, p. 47-48.

4. Abdelwahab Meddeb, Aya dans les villes, Montpellier, Fata Morgana, 1999.

5. Cf. Gérard Genette, « D’un récit baroque », dans Figures II, Paris, Seuil, 1969, p. 222. Le type d'hérö̈sme à l'œuvre ici correspond à l'état de résistance à l'instrumentalisation, la codification définitive du palais, du corps au même que du corpus. L'urgence est celle qui consiste, à chaque fois, à désenclaver « ce qui fait lieu dans l'espace » (selon l'expression de Jean-Luc Nancy, « Entretien » avec Yvonne Rainer, Rue Descartes, n 44, 2004, p. 76) matériel et/ou immatériel; ce qui fait lieu, $m i$-lieu, « cuisine » et « atelier » sans lesquels l'on se condamne, ici ou là, à une clôture plate, anémique et amnésique.

6. Abdelfattah Kilito, «Portrait de l'avare en héros », dans Les Arabes et l'art du récit. Une étrange familiarité, Arles, Sindbad/Actes Sud, 2009, p. 122. 
pour l'essentiel, l' « échange » au « don », les « lettres » et les « livres », elle s'en sert comme d'une " monnaie » tantôt « vraie », ûmlâ Sâlihâa tantôt « fausse », ûmlâ tâlihâa , empêchant le langage de (s)'essayer (à) de nouvelles « expériences », de nouvelles « recettes ». La poésie, synonyme au départ d'un " corpus démoniaque », la vocation rhétorique devant être la sienne au même titre que celle du corps, contraint à l'évacuation, au rejet de la moindre dépense inutile, le moindre « banquet », terme équivalent en langue arabe de maâdouba, lequel n'est pas sans liens, notons-le, avec $A d a b$ et $A d i ̂ b$, « littérature » et « hommes des lettres ${ }^{8} »$.

Le « héros écrivain », fin cuisinier et gastronome, n'aura de cesse, lui, de « bricoler et de recycler ${ }^{9} »$ des « recettes » dont la subtilité et l'inventivité participent au désenclavement d'un goût archaïque autant que contemporain mais dont la "densité originelle » reste, le plus souvent, refoulée, interdite. La cuisine et/ou la littérature : « accumulation de commencements » (Ponge), surgit, chez Abdelwahab Meddeb, à l'image d'une place dont le moindre « fragment » ne cesse de se ramifier, de s'épaissir. Produisant et recevant, en même temps, ce qu'il nomme ailleurs « don et excès ${ }^{10}$ », la tâche ou mieux : la « tache blanche » (titre du premier fragment ouvrant le recueil susmentionné) de l'atelier à l'œuvre consiste à frotter l' « ancien » et le « neuf », le " mien » et le « sien » en les croisant, en les entremêlant sans fins. Aucune antériorité ni

7. Abdallah Bounfour, «Allûgha, Al'adab, Attârîkh », Attakâfa Al-Jadidâ, nº 10-11, 1978, p. 72-78. 8. Voici comment le poéticien, relevant le passage du dogmatique au rhétorique, souligne la persistance d'un même, ou presque, parti pris : l'existence d'une volonté de pouvoir qui, allant de pair avec ce qui se prescrit tantôt au nom d'une «norme sacrée » tantôt au nom de « codes », peu ou prou, « techniques » et « sécularisés », reconduit la même réticence, voire la même méfiance au sujet du signifiant, et ce, quelles qu'en soient la substance et la texture, verbale, factuelle ou iconique : «En arabe, le poète se dit shâ'ir : cela signifie qu'il sait ce que le commun des mortels ne sait pas. D'où tient-il son savoir? D'un démon inspirateur qui lui est personnellement attaché et qui lui souffle ses vers. L'incompatibilité entre la prophétie et la poésie tient à l'origine de l'inspiration, divine dans la première, démoniaque dans la seconde [...]. Après la constitution de l'empire arabe et les grands bouleversements culturels qui l'ont accompagnée, cette figure déserte la scène et n'est plus qu'un souvenir littéraire, un thème ludique évoqué à l'occasion, non sans humour, dans des textes en prose comme L'Épître des génies inspirateurs d'un Ibn Shuhayd. Le poète n'est plus considéré comme le porte-parole d'un démon et n'est plus dès lors dépositaire d'un savoir surnaturel. [...]. Cette évolution est due à la diffusion de l'écriture, à la codification de la lexicographie, de la grammaire, de la métrique, des tropes et figures de style, ainsi qu'au développement de la critique poétique. Le poète partage son savoir avec les tenants de ces diverses disciplines. À partir de ce moment, l'accent est mis sur la maîtrise de la technique poétique et l'habileté de la composition; à l'image du poète inspiré par une force occulte se substitue celle du poète appliquant les recettes d'un $\operatorname{art}(\sin \hat{a}$ ' $a)$. [...] Le monde est quelque peu désenchanté après la disparition des démons inspirateurs, mais on essaie d'y remédier par les splendeurs de la rhétorique » (Abdelfattah Kilito, op. cit., p. 15-16).

9. Gérard Genette, Palimpseste, Paris, Seuil, 1998.

10. Abdelwahab Meddeb, «L'excès et le don », Kalima. Regards sur la culture marocaine, $\mathrm{n}^{\circ} 1,1988$, p. 61-64. 
postérité n'y restent immaculée, séparée et autosuffisante. Travail de mise en espace, de mise en cuisine, l'atelier, agit au sens d'une interface permanente entre « sujet», « mots » et « mets ». Il s'agit, à chaque fois, d'un tout poreux, inachevé, et qui plus est - réciprocité, réversibilité et alternance obligent - qui s'arrache à tout binarisme du type mémoire/oubli, sacré/profane condamanant l'« atelier » en même temps que la « cuisine » à des séparations et des coalescences dites sans failles. Á quelque niveau que l'on se situe, nous assistons au déploiement d'un incessant, insatiable, je(u) de traits d'union - littéralement : interruption et intersections -, dégageant le $m i$-lieu qu'est la « table » et/ou la " bibliothèque » en tant que " place » en devenir, palimpseste ou architec(x) ture, prouvant, si besoin est, que la non-place érigée et prescrite par le dogme et la rhétorique s'avère constamment ébréchée, dés-emmurée ${ }^{11}$.

Dès l'incipit du premier roman : Talismano, pointe ce qui se joue, se rejoue et se déjoue depuis toujours, à savoir l'urgence d'un talisman, d'une thérapie destinée à parer au risque réel d'un corps définitivement "séparé de son énergie » (p. 8), état exigeant d' « injecter sang déverser pleurs ne serait-ce qu'en ces heures mourantes » (p. 9). En effet, d'entrée de jeu, la « main », qualifiée de : « composante de [1]'être » (p. 32), est ce qui ne cesse de faire face au risque de se voir : " cramée, paralysée et l'idée traînant entre les berges : l'idée qui parfois s'emprisonne et paralyse les doigts » (ibidem). Combinaison et déclinaison faites d'au moins quatre langues : grec, arabe, latin, italien : tesma / talsam / telesma / talisman, le titre du " roman » déploie, dès le seuil un jeu de substitutions où, entre autres, magie, exorcisme, signes, graphes, lettres, images, etc., se dégage la présence irréductible d'une ambivalence, une arborescence et une cosmogonie. Lors d'un arrêt en Égypte, la rencontre du « héros écrivain » avec un :

faiseur de talismans et autres écritures, tapis de sable, commissures écumantes, édifiant sur le tracé du sol des tablettes de correspondances astrologiques, lisant dans ma main les composantes de mon être, me regardant avec une espèce de fierté détachée, il ne me communique pourtant rien de ses découvertes comme s'il avait la certitude que je demeure à l'abri des périls, comme si les embûches et fondrières ne valaient pas révélation. (p. 31-32)

Une telle rencontre, occasion d'un portrait autant que d'un autoportrait, dit la passion de celui qui, croisant, entremêlant, comme c'est le cas ici, médecine, astrologie et écriture, ne cessera de « prendre par une main refaite propre, spécifique » un objet, tel le talisman, qui, tout en étant à portée de

11. Force est de souligner qu'aussi bien la culture «populaire » (voir plus loin) : orale, carnavalesque, que la culture « savante », écrite, échappent, ou du moins, résistent, au dit dogmatique et au dit rhétorique : la première via, entre autres, la " poésie bachique », la seconde, à travers un anthropologique fait de dons et d'excés : un rite comme les moussems, pélerinages, ne va pas sans une part de « rudiments esthétiques », une hybridité telle que la séparation et la coalescence sur lesquelles veillent de près « normes » et « codes » établis se voient effectivement ébréchés, dés-emmurés. 
mano, reste littéralement imprenable : « Malheur! L'objet enchanté, rebelle à la prise » (ibidem), lequel est sans doute l'autre nom de la « littérature » et/ou de la « cuisine » au sens où les deux sont constamment en quête de la matière-mémoire : la proximité lointaine et le lointain si proche indispensable au processus sans fin qu'est le « bricolage de l'incurable ${ }^{12}$ ».

Le même type de tension surgit rien moins qu'au cœur de l'anthroponyme, le nom d'auteur, Abdel-wahab (« Serviteur du Donateur ») Meddeb, objet d'un décryptage " archéologique », non sans résonance avec ce qui se joue ici :

Le nom m'épèle : du prénom, servitude déjà dite don, y a rien; non que tu sois pingre ou dépensier à outrance : tes yeux seuls fermentent le don malgré l'apparence farouche et la mélancolie primesautière. Le nom propre change : à l'origine $M u$ 'addib, maître, enseignant, ordonnant le savoir en vue de son bon usage social, dispensateur d'adab, entraînant par le nom l'officielle socialité, à former les bureaucrates et autres exécutants des pouvoirs arabes ${ }^{13}$.

Oscillant entre don et servitude, le nom propre relève, peu ou prou, d'un arbitraire, un im-propre tout aussi « rebelle à la prise » qu'est toute socialité établie : le commentaire méta-anthroponymique ci-dessus relève en somme l'existence d'une autre forme de dépense ruinant, ou du moins, troublant toute séparation et toute coalescence identitaire définitive entre texte et paratexte (ou mieux : transtexte), auteur et écrivain, œuvre et vie ou encore don et échange, table et bibliothèque.

En effet, agie et agissante, la cuisine dans Talismano fait partie d'un atelier favorisant un incessant je(u) d'interactions fertiles et nécessaires à la conception et à la réalisation d'un talisman à même de résister au risque réel qu'est tout binarisme, cause autant qu'effet, d'anémie, d'amnésie. Le passage d'une ville à l'autre, d'une époque à l'autre auquel nous assistons dans ce premier roman, est, à l'instar de la cyclothymie des oiseaux migrateurs faisant pont entre les deux rives de la Méditerranée : toile de fond de l'ouvrage, une incessante tentative de fabrication d'une poé-thérapeu-tique contre le risque de disparition et d'oubli irréversibles d'une identité-altérité. Ce dont il s'agit est d'un permanent effort d'être au plus près d'un travail de raccordement

12. Depuis la série de toiles de Ahmed Charkaoui intitulée : «Talisman » (1965-1966), celles de Mounir Fatmi intitulées : «Antibiotique » (Mounir Fatmi, " Médecines douces », dans Suites marocaines, Paris, Revue Noire, coll. «Art Soleil», 1999, p. 23-27) et l'installation à plusieurs variantes de Mohammed El Baz intitulée : « Bricolage de l'incurable » (Mohamed Rachedi, Interférences de l'art contemporain : références marocaines de l'art contemporain, Amiens, Le-RARE, 2005, p. 70-84), force est de relever la persévérance d'un type de quête où arts - y compris bien évidemment « l'art de la table »- et médecine conjuguent leurs efforts afin de s'acheminer vers la «fabrication » d'un corps et/ou d'un corpus dégagé de l'illusion d'une « recette » et/ou d'une « thérapie » dite « définitive ».

13. Abdelwahab Meddeb, op. cit., p. 218. 
passant outre normes et codes dits définitifs : telle est la raison d'être de celui qui, accompagnant la continuelle procession des révoltés de la ville de Tunis, pointe la figure emblématique de ceux et celles qui « défendent leur ville par la pluralité de [leurs] énergies en résistance » (ibid.), le « peuple de révoltés, une colonie de juifs et de réfugiés andalous du quartier l'Ariana » (p. 88) et qui plus est, se livrant, lors d'une pause, à la mise à feu de leur « idole » dont les « organes suturés font voltiger une singulière odeur de symbole » (p. 90), organisent un rite à la fois grave et festif, normatif et transgressif, où la nourriture la plus variée est, à la tombée de la nuit, servie par de « malicieuses cuisinières » invitant à « manger et boire » les « vieillards » qui « ressuscitent » et voient leur « goût redressé » (p. 89); « ogresses et démons se mettent ainsi au service du peuple en fête » et les « sœurs habilitées à raconter », " servantes de Shahrazade », ne manquent pas, elles, de dire la force d'une culture orale, carnavalesque, où « les femmes s'imposent; ordonnent et condimentent les plaisirs » (ibid.) de sorte que :

beaucoup de vieillards, au passé religieux, à la recherche éculée du rachat, retour de l'âge, ne tiennent pas à boire, vin fort et pétillant sensibilisant larmes à nombre de malicieuses cuisinières, Qamar, Bakhta, Hallûma, Sâlha, Mbârka, toutes prêtes à s'arranger histoire de sexe, à vivre près des chaudrons les cris les plus longs de la jouissance répétée goût d'épices, mains et vagins imprégnés par les sept condiments : basilic, girofle, cumin, poivre, safran, gingembre, cannelle, à doser langue en collaborant avec les sœurs habilitées à raconter, à broder sujets tabous, servantes de Shahrazade, vieilleries à nous endormir rêvant prince et trésor, puits et caves, ogresses et démons à mettre au service du peuple en fête, à participer au profit de l'oubli de soi à la connivence de haute teneur de la femme et de l'enfant tout en laissant intact un semblant de pouvoir à l'homme berné par l'apparente non-intervention, au point de se découvrir dupe, victime de l'opulence même intégrée source inefficiente de pouvoir. (ibid.)

Faisant trait d'union entre religion, gastronomie, érotisme, musique et conte, ce « festin au cent plats » est une occasion où l'on voit s'interférer, s'entremêler en termes inextricables, réversibles, sacré et profane, nord et sud, antiquité et contemporanéité :

N'était-ce en vrai et sans nostalgie la fête du Transtevere remise à Tunis, reflet pauvre de Rome? Qu'y manque-t-il sinon un degré autre de l'ostentation et de la richesse, sinon la tradition des sculptures et fontaines monumentales, eau musique des nuits abasourdies par la chaleur d'été, sinon fresques et mosaïques célébrant sur les façades les paysages du Mythe, Jérusalem céleste, Sodome, conquête des continents? Mais les réseaux culinaires, érotiques, spectaculaires et leurs imbrications tiennent d'une même topique productrice d'une geste similaire par l'humour, par le discours, par la valeur dès que la parole s'infeste par l'haleine provocante des gouapes [...]. (p. 197)

Rite idolâtre et iconoclaste, la fête et la procession, se révèlent topique d'avant tout emmurement dogmatique et rhétorique, elles sont l'équivalent 
d'un véritable acte de dé-place-ment de savoirs et de saveurs, d'un jeu d'emboîtements paradigmatiques dégagé de la moindre hiérarchie.

Revisitant le même type de $m i$-lieu, le récit intitulé : « Retour à Fès » du recueil Aya dans les villes dit, lui aussi, le risque qu'est le ressassement mémoriel, la « répétition » réduite à du « ra $(\mathrm{e}) \mathrm{b}(\mathrm{m})$ âchage ». Lequel, évitant la déambulation, le cheminement vers une sensation d'enfance, première, comme le fait le « héros écrivain », accompagné d'Aya, s'empêche, au fond, de vivre l'expérience de la lointaine et si proche matière-mémoire :

L'odeur de la fève fraîche extrait de vielles images, Aya se voit enfant de trois ans lorsqu'elle jouait à aider sa mère à écosser les légumes verts, dans la cuisine dont elle ne conserve aucune image, dit-elle, elle ne la situe qu'en recourant à l'évocation des aînés, lucarne qui donne sur les remparts rouges, protégeant de l'arrière-pays, entre l'océan et le désert, serait-ce, lui dis-je, vestiges métamorphosés en souvenirs à force d'avoir été rabâchés par d'autres témoins? Il n'empêche que le goût de la graine dont je reçois l'odeur m'envahit le palais et me déporte vers une halte de mes pérégrinations italiennes, dans une auberge toscane où j'avais croqué, en guise d'apéritif, des fèves crues accompagnées de grana, vin blanc local, vif, fougueux et qui ne voyage pas ${ }^{14}$.

Le trait d'union liant-séparant l'« odeur de la fève fraîche » et les souvenirs permet de rendre possible, effectif, le cheminement vers l'arrièrepays dégagé du voile des récits d'origine dont le régime de temporalité chrono-logique à l'abri du chaos que sont les saveurs et les couleurs, fait que ladite origine, si origine il $\mathrm{y} a, \mathrm{~s}$ 'avère réduite à un lieu commun, un non-lieu, où le « je » et « elle » tentent, eux, de s'approprier la place qui est la leur entre océan et désert, mémoire et éclats, retrouvailles et égarements.

Le risque d'amnésie (anémie) ne cessant néanmoins d'être réel, permanent, cela exige du « héros écrivain », dans le sillage de l'après-Talismano, l'urgence de faire face à une véritable pathologie : «Entre la puissance qui voit en vous, écrit Meddeb dans son deuxième roman Phantasia, un sousêtre et l'humiliation qui déifie la vengeance; entre les moyens guerriers qui vous écrasent et le harcèlement du faible mû par la haine aveugle, s'enveloppe une monstrueuse nodosité qu'il m'appartient de trancher ${ }^{15}$. » Se frayer un chemin loin de l'impasse qu'est cette double clôture vouant le sujet et son moi à n'être qu'un « sous-être » : véritable « nodosité », n'est-ce pas, au fond, une « affaire » de lecture, d'interprétation autant que de greffes sur greffes? Qu'il s'agisse de Talismano ou de Talismano bis, le travail dont il s'agit est celui d'une cuisine autant que d'une clinique où s'opère, selon la logique d'une quête à la fois désenchantée et heureuse, l'acte de ce qui reste inévitablement acte expérimental, « bricolage de l'incurable».

Dans un éditorial de la revue Dédale consacré à la thématique de « La venue de l'étranger », intitulé : «Les fins de l'étranger », l'auteur diagnostique

14. Aya dans les villes, op. cit., p. 116.

15. Abdelwahab Meddeb, Phantasia, Paris, Sindbad, 1989, p. 118. 
un autre type de maladie qu'il nomme le « littéralisme ${ }^{16}$ » empêchant de voir «l'extension du possible », entravant même la circulation du (des) « sens » :

Si en effet, le prophète Muhammad clôt la série prophétique, l'imam'Alî ouvre la descendance imamologique. La prophétie apporte la lettre, mais une telle lettre serait lettre morte si elle n'était pas animée par le sens qu'en tire l'imam. La lettre qui est révélée au prophète serait le noyau de l'amende; et l'imam se saisit d'un tel noyau, le soumet à la meule de l'interprétation pour en extraire l'huile. Cette disposition ouvre le champ de la lettre, lequel était destiné à la clôture ${ }^{17}$.

Réduire, aplatir la texture du signifiant, qu'il s'agisse d'un mot ou d'une amande, cela empêche de voir le Livre relancé dans de nouveaux mi-lieux, de nouveaux con-textes. La fidélité à la lettre, réduite à une surface lisse, voilà ce à quoi s'emploie et condamne le « littéralisme ». Quelles qu'en soient la nature et la substance, le signifiant et sa capacité d'être et autonome et dépendant, cela ne relève-t-il pas du tact, du geste et de la force haptique capable d'en extraire aussi bien les « sens » que les « essences »? Dans l'essai Les Fins de l'étranger cité ci-dessus, le culinaire, foyer de l'ambivalence par excellence, agit en tant que lieu qui, circonscrit et ouvert, permet l'expérience à la fois de l'appartenance et de la dés-appartenance :

«Comme lorsque, dans le non-lieu de l'étranger, je me suis inventé une demeure pour accueillir des autochtones férus de leur tradition, stricte dans la règle qui gouverne l'association des (breuvages) et des mets. Nous fûmes les hôtes de ces amis qui ont la vertu de dire avec exactitude le nom de l'étranger (ils auront toujours ma tacite gratitude, ceux-là qui ont le don d'honorer le nom barbare); avec eux, nous avons accompagné un couscous fassi d'un Sauternes, un $1^{\text {er }}$ cru château La Tour Blanche 1976. L'accord est absolu et sollicite tous les sens : d'abord les yeux s'égarent et se retrouvent à travers les parcelles de la palette lorsque le breuvage jaune paille éparpille ses reflets sur les teintes de la semoule blanche rehaussé par la cannelle qui l'imprègne comme par la sauce qui l'imbibe, laquelle est à son tour colorée par la fleur de safran; ensuite le nez butine entre les bouquets de coing, de vanille, d'amande citronnée et de miel d'acacia qui émanent du vin pour se conjoindre au miel qui avait fusionné avec les oignons et les raisins secs, couche caramélisée qui couvre le monticule de graine et les morceaux de poulet fermier qui s'y nichent; enfin en bouche les suavités se confirment, se complètent, se prolongent, s'équilibrent entre le rôti du vin et le rôti du poulet, entre le gras et l'amertume acidulée qui se fondent dans le cycle des rondeurs qu'esquissent l'un et l'autre miel. C'est comme si le plat africain et le breuvage girondin étaient faits l'un pour l'autre », s'exclame l'un de mes convives, surpris en son autochtonie par la réussite de l'audace.

Outre son audace indéniable, imprévisible, la recette ci-dessus, synonyme d'un « champ de "création" qui s'élargit lorsqu'on infléchit la 
combinaison du sec et du frais vers une orientation marocaine, ou qu'on imprime aux fronts de l'aigre et du doux le flux d'une énergie chinoise » (p. 13), agit en tant que proposition qui, passant outre le binarisme du genre : sucré/salé, licite/illicite, s'arrache, sans détours, au ghetto de la pureté et de l'autochtonie repliées sur elles-mêmes.

Dans «Une journée du monde », court récit-essai d'autoportrait, re-commence autrement le même rapport de tension entre gastronomie et quête d'identité-altérité :

Le soir, nous recevons des amis et collègues égyptiens. Dans l'amitié, nous circulons dans le labyrinthe des langues; Sonalah cherche à communiquer en allemand avec Hind; Amina teste ses rudiments d'arabe littéraire avec Leila; Nabil retrouve ses expressions de Broklyn en parlant avec l'étudiante américaine. Nous partageons un grand saumon frais accompagné d'un blanc du Jura, un Château-Chalon dont la pointe brûle la langue au bord d'un goût acide et sec qui le rapproche étrangement du Xérès. Et son bouquet reste modéré et bref au point de ne pas atténuer à la délicatesse du poisson. Toutefois, telle association est accidentelle et elle reste téméraire ${ }^{18}$.

Le pouvoir qu'ont les mets d'associer librement goûts et saveurs, cela rend possible, viable, l'expérience de conciliation des « contraires » exacerbés par les intolérances. Dès lors qu'elle est l'œuvre d'un jeu d'extension permanente, la cuisine permet de faire prendre, fût-ce le temps d'une soirée, un autre type de cohabitation, de négociation. Les recettes, notamment celles qui ne se consomment plus uniquement « entre soi », participent à réunir, à « fusionner » les convives de divers pays, divers continents. La recette-proposition du « donateur », Al Wahâb est enfin celle qui re-commence, toujours avec le même appétit, la quête d'une « médecine de l'âme ${ }^{19}$ » : produit et matrice d'un jeu de " bricolage et de recyclage » rompant avec le local autosuffisant au même titre qu'avec le global et son universel conquérant.

Non sans faire écho à une telle quête talismanique, le rapport au gastronomique et au culinaire à l'échelle de la littérature maghrébine est aujourd'hui « lieu » d'enjeux sans doute moins « archéologiques » mais tout aussi décisifs, insistants. Face à l'usage qu'en fait un discours béatement folklorique, l'auteur de Partir, Tahar Ben Jelloun, écrit dans un ouvrage collectif intitulé Comment peut-on être marocain? : "Commençons par une dose importante d'autocritique; désigner les facteurs déclencheurs de cette mauvaise image [du pays]; en finir avec le système "invitation pastilla-poulet aux citrons confits, agneau aux pruneaux et amande" et le

18. Abdelwahab Meddeb, «Une journée du monde », Le Nouvel Observateur (Album anniversaire 1964-1994), Hors Série no 22-23-24, 1994, p. 170.

19. Abdelwahab Meddeb, « Médecine de l'âme », Entretien avec Amira Ben Youssef, Le Renouveau, Tunis, 1993, nº 1511. 
bruit fait autour de la fameuse "hospitalité marocaine" 20 ». Face au culinaire et au gastronomique rendus cliché identitaire se dresse le parti pris, serein et intraitable, d'un essayiste qui, muni d'un " goût dans la bouche », aborde la question de façon active, créative, où les mots et les mets restent liés les uns aux autres selon une logique de mixage, de métissage :

Il faut, écrit Khalid Zekri dans le même ouvrage, relativiser la peur d'uniformisation qu'engendre la globalisation. Il est certes aisé de montrer l'uniformisation matérielle qu'implique la globalisation. Mais qu'en est-il de l'aspect existentiel, immatériel de la culture dans le contexte de la globalisation? Je pense que cette uniformisation matérielle progressive connait des déclinaisons variables selon l'usage que chaque culture (locale) en fait. Prenons l'exemple du McDonald's et de la McDonaldisation du monde. Cette chaîne de fast-food s'adapte à la demande et aux habitudes " culinaires » de chaque culture dans laquelle elle décide d'avoir une filière. Au Maroc, par exemple, sur les menus du McDonald's se trouve une variation du McTajine. Peut-être avec un peu plus d'imagination, et pour répondre à l'attente d'une population versée dans une négociation forcée entre tradition et modernité, nous pourrons avoir un menu McPastilla ou un petit-déjeuner McMlawi avec Big, Moyen ou Petit Thé ${ }^{21}$.

Il y a là une inter-zone propice à une négociation sans fins. Foyer de recettes assumant les risques autant que les chances du frottage aux sociétés et aux cultures " créolisées et dé-purifiées ${ }^{22}$ ", la nouvelle-ancienne cuisine rend sinon impossible du moins difficile toute ligne de démarcation catégorique entre mien et sien, copie et original, centre et périphérie.

Sans doute, la cuisine et/ou la littérature chez Abdelwahab Meddeb est-elle seuils et corridors d'un atelier qui, s'élargissant, expérimentant, en permanence, est celui du " passeur de cultures », le " contrebandier ${ }^{23}$ »" qui dé-re-façonne inlassablement le moindre prototype dit clos et définitif. Pratique et théorique, trans-frontalier et trans-générique, l'atelier à l'œuvre dans le livre inachevé veille de près sur le processus de fabrication d'une poé-thérapeu-tique résistant aux binarismes et leurs recettes-cures exclusives, miraculeuses.

\section{Abdelkrim Chiguer Université de Meknès - Équipe d'Études Culturelles et Postcoloniales}

20. Tahar Ben Jelloun, dans Abdessalam Cheddadi (dir.), Comment peut-on être Marocain?, Rabat, Éd. Maison des Arts, des Sciences et des Lettres, 2009, p. 35-45, p. 45.

21. Khalid Zekri, dans, Abdessalam Cheddadi (dir.), op. cit., 189-201, p. 198.

22. Gérard Genette, «L'Art en question », dans Figures V, Paris, Seuil, 2002.

23. Abdelwahab Meddeb, "Chemins de contrebandes ", dans L'Exil occidental, Paris, Albin Michel, 2005. 